\title{
The Defect Detection of Fibre Boards Gluing System Based on TRIZ
}

\author{
Huiling $\mathrm{Yu}^{1}$, Guangsheng $\mathrm{Chen}^{1}$, Congchun $\mathrm{Xu}^{2}$, and Delin $\mathrm{Fan}^{1}$ \\ ${ }^{1}$ Northeast Forestry University \\ No. 26,Hexing Road,Harbin, 150040,P.R. China \\ ${ }^{2}$ Heilongjiang Province productivity promotion center \\ No. 74,Yinhang Street,Harbin, 150001,P.R. China \\ dlfan33 @yahoo.com.cn
}

\begin{abstract}
Defect signal is always a problem that interferes with the operation of fibre board gluing control system. Complex problem analysis and solving are an important evolutionary direction of TRIZ. This research creates a defect diagnosis methodology by synthesizing the Theory of Inventive Problem Solving (TRIZ) and a Possibilistic C-Means (PCM) improved Support Vector Data Description (SVDD) method. Applied TRIZ to identify core root causes and confirm contradictions to classify the system defect. A complex problem solving model integrating SVDD and PCM in TRIZ frame is put forward, a case shows the application of this model.
\end{abstract}

Keywords: Theory of inventive problem solving (TRIZ), 40 Inventive Principles, defect diagnosis, support vector data description.

\section{Introduction}

Theory of Inventive Problem Solving (TRIZ in its Russian abbreviation) is an analytical approach to creative engineering. It has been developed in the former Soviet Union by Genrich Altshuller. TRIZ is an algorithmic methodology which helps break psychological inertia in problem solving. It also provides engineers with powerful algorithmic approaches to formulate, analyze, and solve complex engineering problems, as well as to use objective Laws of Evolution of Technological Systems for directed development of next generation products and processes. The main postulate of TRIZ is as follows: Evolution of engineering systems is not a random process, but is governed by certain objective laws. Altshuller formulated eight laws of evolution of engineering systems. These laws can be utilized for conscious engineering system development briefly described below. Altshuller analyzed about 400,000 invention descriptions from different fields of engineering. The most effective solutions were selected and examined to reveal the objective laws (trends) of evolution of engineering systems. The evaluation of the solution's effectiveness was based on the concept of engineering contradiction: A problem becomes a creative one when an attempt to improve system's parameters by conventional means leads to deterioration of other parameters generates an engineering contradiction[1][2]. 
TRIZ was born as a problem-solving tool based on a systemic view of the technological world. The primary idea behind TRIZ approach to solving problems is that information about a specific problem must be first generalized, a solution concept has to be generated, and then the concept should be specialized in terms of a feasible solution. Traditional problem-solving methods aim to find a specific solution to a specific problem often using brainstorming or trial and error processes. In contrast, TRIZ translates the specific problem into an abstract problem or model of the problem and then to an abstract solution or model of the solution. Figure 1 depicts this strategy.

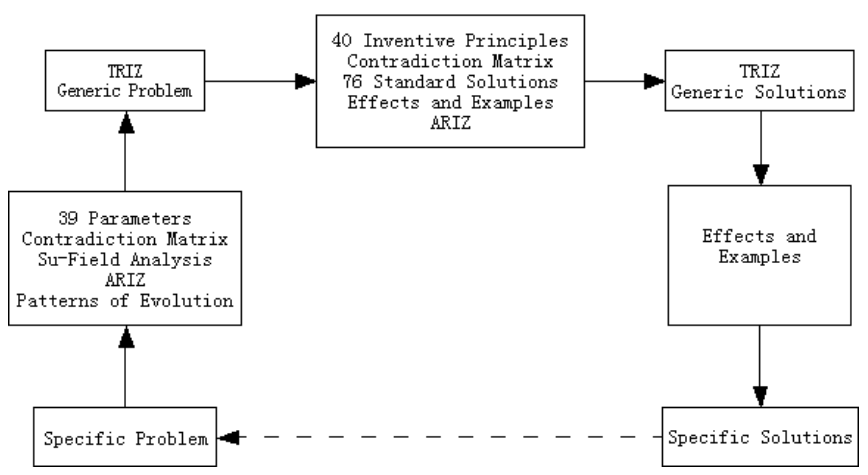

Fig. 1. TRIZ model for problem solving

TRIZ has been successfully applied in a number of industries to structure the innovation process. Mixing and supplying glue technology of particleboard is one of the key technologies on particleboard production line, although the investment of the entire section of all investment in whole line just accounted for $3 \%$ to $6 \%$, but it makes significant influence on whole line production. In the same process condition, excessive glue application will lead to high moisture content of particleboard, which can easily result in bubbling phenomenon and increased production cost at the same time; if glue application is insufficient, the plasticity of particle will be small, the bending strength and plane tensile strength of particleboard will decrease with the reduction of glue application, which lead to the decline in the quality or substandard products.

Gluing is one of the key steps in the manufacture of fibre board, and gluing control system directly affects product quality and costs. Improper proportion of the glue will lead to the degradation of the physical properties of fibre boards. In the gluing procedure, the quantity of glue should be kept with the change of fibre, and previous researches have proposed a series of optimal algorithms to control the non-linear model of gluing system, but the defect signals, which always interferes the fibreboard gluing control system, is ignored. Existing innovation methods in control field are mainly genetic algorithm or fuzzy-PID. In this paper, a systematic approach to build a method integrated TRIZ and SVDD for gluing system control strategies was proposed. 


\section{Background of TRIZ}

Theory of Inventive Problem Solving (TRIZ in its Russian abbreviation) has been developed in the former Soviet Union by Genrich Altshuller, starting in the fifties. The main postulate of TRIZ is as follows: Evolution of engineering systems is not a random process, but is governed by certain objective laws. Altshuller analyzed about 400,000 invention descriptions from different fields of engineering.

The difference between control strategy and numerous patents on which TRIZ was based makes some inventive principles, universal parameters and general solutions in TRIZ hard to get correspondences in control theories directly. Applying TRIZ into an integrated control system should include some basic parts, for example, laws and routes of control strategies evolution, levels of invention of control strategies, contradictions and their types in control strategy design problems.

\subsection{The Ideal Design with No Harmful Functions}

\subsubsection{Technical System Describe}

Gluing is the key process for the particleboard production, the adhesive which distributed evenly in the debris on the surface will have a direct impact on the quality of the finished product. We considered the factors impacting on the particleboard quality in the process of system analysis and got the sub-system and the super-system. We defined the Operational Zone(OZ) and the Operational Time(OT) then using the three-dimension analysis to get the Route Course(RC). To control the flow of the glue and the rotate speed of the pump according to the weight of the fibre.

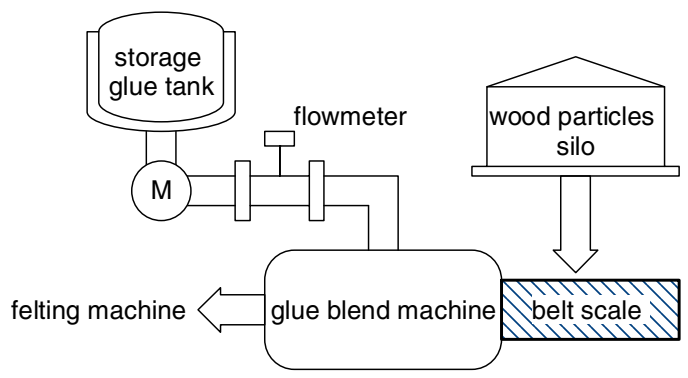

Fig. 2. Particleboard supplying glue system

\subsubsection{Definition of Glue Dosing System}

A system can be thought of as "a group of interacting, interrelated, and interdependent components that form a complex and united whole. Systems are almost always defined with respect to a specific purpose within a larger system. The system of supplying glue section in particleboard production line is composed of a glue storage tank, measuring devices, glue pumps, pipes and other components, divided into surface and core layer applying glue, with the same process and the symmetry structure. To solve the existed problem, the following supersystem in which the system resides were considered, such as air, the shape of flake, the pipelines and the density of the flake etc. 


\subsection{Functional Analysis of the Glue Dosing System}

When analyzing this case, the first step was to describe the components in and around the system; the second detailing the useful relationships between each pair of components; the third then describing the negative (harmful, insufficient, missing or excessive) function present in the system.

The primary useful function of the glue dosing system is to measure flow accurately. Through the proportioned glue is stored in glue storage tank in accordance with the timing, supplying glue process is that shavings is sprayed by the corresponding glue mixture which is pumped from the two glue storage tank separately according to the weight of surface particle (thin stuff)and the weight of core layer and particle weight (coarse material), the particles is transported by Electronic belt scale, particle flow is measured precisely by rotary encoder, and glue content is measured by electromagnetic flow transmitter and load sensors, and then supplying glue steadily is realized though the method that using frequency converter to control the supplying glue pump.

\subsubsection{Define the System Boundaries and Environment}

TRIZ is a scientific methodology for defining, analyzing and formulating solutions to difficult problems usually of a technical nature. This research includes other systems nearby the supersystem: forming machine, hot press and sanding machine.

\subsubsection{Conditions around the System and Its Supersystem}

Mixing and supplying glue system is a complex system with the characteristics that great time-delay, great inertia, multiple disturbance, nonlinear and time variation, and these characteristics make supplying control more difficult.

\subsection{Define Available Resources}

Since glue cost is high and the supplying glue control has a direct impact on hot pressing results of particleboard, the large time delay characteristic of supplying glue system affects product quality seriously. In addition, as the factors that electromagnetic interference, pressure variation inside and outside the pipe, mutations of shavings flow, failure shutdown etc exist in the control system during the supplying glue process, the traditional PID controller is difficult to achieve the required control quality of the system and manual intervention is often required.

Resources refer to anything in or around a system which is not being used to its maximum potential. TRIZ looks for resources of innovation in the system itself not in the individuals or the organization surrounding it, so we can manage and guide the processes of value maximizing by problem solving. Any physical object with mass or that occupies space and that potentially can be used to improve a system calls substances. Includes Substance Resources: glue, flake, air etc, Space Resources: the length of the pipe, Time Resources: the time of flowing through the pipe, Information Resources: the flake's shape and density; Fields is defined as the energy needed for the interaction of substances within a system that can potentially be used to improve the system. Field resources: Chemical Field, electrical Field, mechanical Field. 


\subsection{The Contradiction in Technical System as a Basis of Innovation}

The evaluation of the solutions' effectiveness was based on the concept of engineer contradiction: A problem becomes a creative one when an attempt to improve system's parameters by conventional means leads to deterioration of other parameters.

In control system, requirements to rise time and accuracy usually can't be satisfied simultaneously with a single control strategy. It would compose a technical contradiction and physical contradiction. From the standpoint of TRIZ, solving a problem means overcoming this contradiction, or satisfying all conflicting requirements. 40 inventive principles (classes) of TRIZ are one of the less widely used innovation tools. Its have been the basis of the study for innovations in mechanism system. TRIZ researchers have configured a tool called a Contradiction Matrix. The contradiction table is a $39 \times 39$ matrix of all the characteristics on the rows and columns. To use it, first look up the character you wish to improve and go to its appropriate row. Next, locate the column of the characteristic that degrades as a result. Where the row and column intersect are numbers that correspond to the 40 inventive principles.

Table 1. Contradiction matrix table

\begin{tabular}{|c|c|c|c|c|c|}
\hline \multirow{8}{*}{ 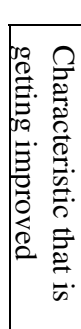 } & \multicolumn{5}{|c|}{ Characteristic that is getting worse } \\
\hline & \multirow{2}{*}{\multicolumn{2}{|c|}{ Engineering Parameters }} & 35 & 36 & 37 \\
\hline & & & $\begin{array}{l}\text { Adaptability } \\
\text { or Versatility }\end{array}$ & $\begin{array}{l}\text { Device } \\
\text { Complexity }\end{array}$ & $\begin{array}{l}\text { Difficulty of } \\
\text { Detecting }\end{array}$ \\
\hline & \multicolumn{5}{|c|}{23 Loss of Substance } \\
\hline & 24 & \multicolumn{4}{|l|}{ Loss of Information } \\
\hline & 25 & \multicolumn{4}{|l|}{ Waste of Time } \\
\hline & 26 & \multicolumn{2}{|l|}{ Amount of Substance } & \multirow{2}{*}{\multicolumn{2}{|c|}{$\begin{array}{l}3,13 \\
27,10\end{array}$}} \\
\hline & & & & & \\
\hline & 27 & \multicolumn{4}{|l|}{ Reliability } \\
\hline & 28 & \multicolumn{4}{|l|}{ Accuracyof Measurement } \\
\hline
\end{tabular}

The next step is to identify the standard and/or proposed contradictions in the case. In the supplying glue system, we found a main contradiction for the Macro-level and Micro-level that we wish to improve from the row 26 amount of substance, thus the undesired result — the characteristic that degrades as a result of improving the above feature, the column 36 device complexity. From the standpoint of TRIZ, solving a problem means overcoming this contradiction, or satisfying all conflicting requirements. Based on the matrix they would obtain the solutions as 3 Local Conditions principle, 13 Inversion principle, 27 Disposable Object principle, 10 Preliminary Action principle. With the sequence given in the descending order of usage.

Among the four recommended principles, number 10 (preliminary action) provided feasible ideas to realize our IFR(Ideal Final Result, the evolutionary end-point for a system). Problems that contain contradictions or conflicts can be solved one of two ways: either by making tradeoffs or by stating the objectives in the form of a contradiction and then solving the contradiction. Usually contradictions are resolved 
though trade-offs by finding some compromise between the two requirements. This results in a solution that does not fully satisfy either element. TRIZ, on the other hand, does not attempt to make trade-offs between two requirements in features or functions. Ideality is typically defined as benefits divides by cost and harm.

The last step in the analysis is to identify the principles used in solving the problem and how they were used. Principle 10 preliminary action-Arrange objects so they can go into action in a timely matter and from a convenient position, place each part of the object under conditions most favorable for its operation. Perform before it is needed.

Increasing system dynamism and controllability allows functions to be performed with greater flexibility or variety. We apply the cascade control system design based on predictor in advance. The Smith Predictor will be employed to the control system. Most of the principles suggested from the matrix match the concepts used in the actual solution of the problem.

The paper also considered solving the problem using a substance resource and field. Substance-Field analysis is a TRIZ analytical tool for building functional models for problems related to existing or new technological systems. Substances may be materials, tools, parts, people or environments. Fields may be mechanical, thermal, chemical, electrical or magnetic. 5 classes of 76 standard solutions are offered. According to su-field concept, model of the problem can be represented by $S_{1}$ (pump). To control $S_{1}$, a pair F- $S_{2}$ should be introduced into the system. $S_{2}$ is to transform $\mathrm{F}$ into mechanical field (force) which would act on $S_{1}$.Due to ineffectiveness of mechanical F, it was natural to suggest transition to microlevel which can be realized by use of control using a predictor.

\section{The Detailed Steps of Using Multi-classifier by SVDD}

\subsection{An Overviews on SVDD}

SVDD was developed to solve one-class classification problems, and its main idea is to map data points by means of a nonlinear transformation to a high-dimensional feature space. In the feature space it first find the smallest sphere that contains most of the mapped data points in the feature space, and then mapped back to the data space. In the way above the data can be separated into several components, each enclosing a separate cluster of points [10] [11] [12]. SVDD obtains a boundary around the target data set, and this boundary is used to decide whether new objects are target objects or outliers.

The simplest idea of SVDD defines a hyper sphere around the data and the goal is to minimize the volume of the sphere. The minimum enclosing sphere $\mathrm{S}$ is characterized by its center $a$ and radius $R$ which keeps all the training objects inside its boundary.

The structural error of SVDD is as follows:

$$
\begin{aligned}
F(R, a) & =R^{2} \\
\text { Subject to }\left\|x_{i}-a\right\|^{2} & \leq R^{2}
\end{aligned}
$$


To allow for the possibility of some samples falling outside of the sphere, we can relax the constraints by introducing slack variables $\xi_{i}$, and then the minimization problem can be turned into (3):

$$
\begin{gathered}
F(R, a)=R^{2}+c \sum \xi_{i} \\
\text { Subject to }\left\|x_{i}-a\right\|^{2} \leq R^{2}+\xi_{i}, \quad \xi_{i} \geq 0
\end{gathered}
$$

Where $a$ is the center of the minimum enclosing sphere $S, \xi_{i}$ are slack variables allowing for soft boundaries, the parameter ${ }^{c}$ controls the trade-off between errors and the volume of the description. To solve this problem, the Lagrange multipliers are introduced:

$$
\begin{aligned}
L\left(R, a, \alpha_{i}, \beta_{i}\right)= & R^{2}+c \sum \xi_{i}- \\
& \sum\left(R^{2}+\xi_{i}-\left\|x_{i}-a\right\|^{2} \alpha_{i}-\sum \beta_{i} \xi_{i}\right)
\end{aligned}
$$

By Using the Lagrange multiplier method, the above constrained quadratic optimization problem can be formulated as the Wolfe dual form. To solve the partial derivatives of $L$ about $R, a$ and $\xi_{i}$ we set their derivative value to zero, and then get (6) (7) (8):

$$
\begin{gathered}
\frac{\partial L}{\partial R}=2 R\left(1-\sum \alpha_{i}\right)=0 \Rightarrow \sum \alpha_{i}=1 \\
\frac{\partial L}{\partial a}=2 \sum \alpha_{i}\left(x_{i}-a\right)=0 \Rightarrow a=\sum \alpha_{i} x_{i} \\
\frac{\partial L}{\partial \xi_{i}}=c-\alpha_{i}-\beta_{i}=0 \Rightarrow c=\alpha_{i}+\beta_{i}
\end{gathered}
$$

Taking (6)-(8) into (5), we obtain the dual formulation

$$
L=\sum \alpha_{i}\left(x_{i} \cdot x_{i}\right)-\sum \alpha_{i} \alpha_{j}\left(x_{i} \cdot x_{j}\right)
$$

Subject to $0 \leq \alpha_{i} \leq c, \sum \alpha_{i}=1$

The maximization of (9) gives a set $\left\{\alpha_{i}\right\}$. Objects $x_{i}$ with $\alpha_{i}$ are called the support vectors (SVs) of the description. (7) shows that the center a of the sphere $\mathrm{S}$ is the linear combination of the support vectors.

To decide whether an object $x$ belongs to the target, the distance from the object $x$ to the center of the sphere has to be calculated. The object $x$ is accepted as a target when this distance is smaller than or equal to the radius; otherwise it is beyond the target. Hence, the following condition has to be verified. $R$ is the distance from the center of the sphere a to any support vector $x_{i}$ on the boundary.

It is worth noting that all $\phi$ mappings used in the SV method occur in the form of inner products. This allows us to define a kernel function $\mathrm{K}$, and the nonlinear SVDD 
can be constructed using only the kernel (10), without needing to know the mapping $\phi$ explicitly.

$$
K\left(x_{i} \cdot x_{j}\right)=\left(\phi\left(x_{i}\right) \cdot \phi\left(x_{j}\right)\right)
$$

\subsection{Constructing Multi-classifier Based on SVDD}

We can adopt one-against-all method to construct multi-classifier algorithm [13]. Given a set of $l$ labeled training samples $z=\left\{\left(x_{i}, y_{i}\right)\right\}_{i=1}^{l}$ with a label space $y=\{1,2, \ldots c\}$, we first partition the training samples into c disjoint subsets $\left\{D_{k}\right\}_{k=1}^{c}$, according to their output classes, each given as $D_{k}=\left\{\left(x_{i}, y_{i}\right) \mid y_{i}=k\right\}$. For each subset $D_{k}$, an SVDD is trained by using $D_{k}$ as the target class and the remaining subsets as the negative samples. This leads to $c$ optimal hyper spheres, with each center denoted as $a_{i}$. Using one-against-all method, the label of an input sample is simply predicted as the class where its nearest-neighbor center belongs to, which is given as

$$
y_{z}(x)=\arg \min _{i=1,2, \ldots c}\left\|\phi(x)-a_{i}\right\|
$$

\section{Possibilistic C-Means Algorithm}

The idea of PCM algorithm was originally proposed to overcome the relative membership problem for fuzzy c-means algorithm, and the algorithm has shown the excellent ability to deal with noises and outliers samples. Its basic idea is to relax the constraint and propose a possibilistic method for clustering by minimizing the following object function:

$$
J(U, V)=\sum_{i=1}^{c} \sum_{k=1}^{n} u_{i k}^{m}\left\|x_{k}-v_{i}\right\|^{2}+\sum_{i=1}^{c} \eta_{i} \sum_{k=1}^{n}\left(1-u_{i k}\right)^{m}
$$

where $c$ is the number of clusters, $n$ is the number of data points, $u_{i k}$ is the membership of $x_{k}$ in class $i, m$ is the quantity controlling clustering fuzziness, $v$ is the set of cluster centers, and $\eta_{i}$ are suitable positive numbers.

First we demand that the distances from data points to the prototypes be as low as possible, and then we force the $u_{i k}$ to be as large as possible, thus avoiding the trivial solution. We can use kernel technique to generate kernel PCM.

\section{Example for the Application of the Proposed Method}

\subsection{The Defect Problem of Fibre Board Gluing System}

The structure of fibre board gluing system is shown in figure 3 , which is made up of 3 parts, fibre supply device, glue supply device, and blender. 


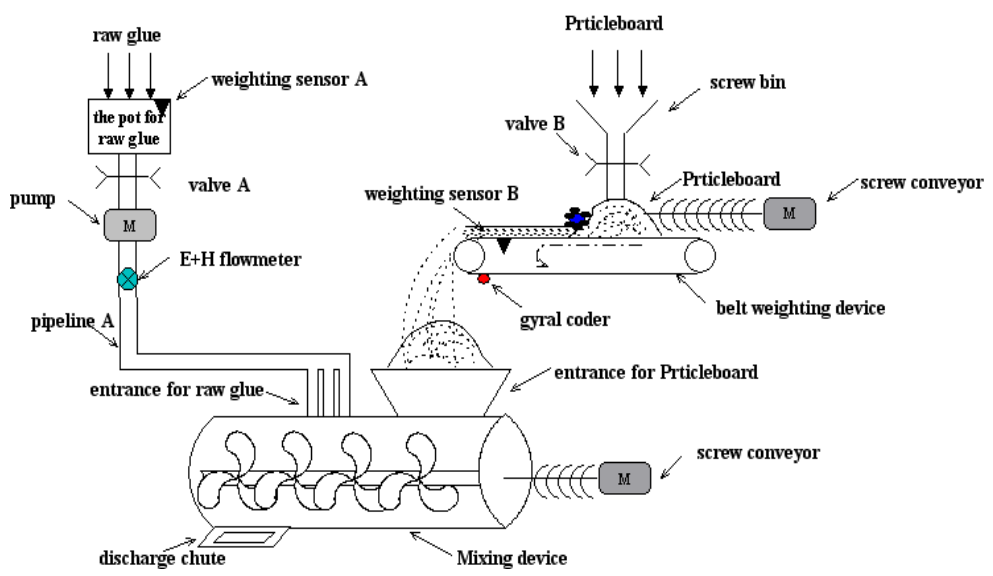

Fig. 3. The structure of fibre board gluing system

In the gluing system, there are usually 6 types of defects. The types are the gluing system is out of control, glue bump does not work well, the glue supply pipeline is blocked, the fibre supply screw is malfunctioned, the blender is blocked, and the flux control of fibre supply does not work normally. In order to map problems to the solution domain, we define the 6 defects as $R_{1}, R_{2}, R_{3}, R_{4}, R_{5}, R_{6}$ respectively, and each symbol matches one type of defect. The symbols and the types of defects are shown in Table 2. Now, TRIZ is used to deal with the above mentioned problem. Accordingly, rich information and insight for idea generation can be achieved by TRIZ technique. When engineers have sufficient training, experience, and practice, they will exploit the potential of TRIZ and search for feasible solutions effectively based on the knowledge base of TRIZ.

Table 2. The types of defect

\begin{tabular}{ll}
\hline symbol & The type of defect \\
\hline R1 & gluing control system \\
R2 & Glue bump \\
R3 & Block of glue supply pipeline \\
R4 & The screw of fibre supply \\
R5 & Block of the blender \\
R6 & Flux control of fibre supply \\
\hline
\end{tabular}

From some workers with experiences with gluing system, we conclude some parameters that can be measured by some sensors. When the defect happens, these parameters change, so we employ these parameters to identify the defect signals. Actually, there are 5 parameters expressing the defect types, they are the glue quantity in the storage which is measured by weight senor, the glue flux in the pipeline measured by $\mathrm{E}+\mathrm{H}$, the fibre quantity detected by weight sensor, and the rotation speed of glue bump and the blender respectively detected by knotmeter. We define the measured data identifying the defect types as $F 1, F 2, F 3, F 4, F 5$. Table 3 gives the matching relationship between the symbol and physical data measured by different sensors. 
Table 3. Physical data recognizing the defect types

\begin{tabular}{clc}
\hline symbol & Physical data & Senor type \\
\hline F1 & The glue quantity in storage & Weight sensor A \\
F2 & The flux in glue pipeline & E+H \\
F3 & The fibre quantity & Weight sensor B \\
F4 & Rotating speed of glue bump & knotmeter \\
F5 & Rotating speed of the blender & knotmeter \\
\hline
\end{tabular}

\subsection{Apply the SVDD Method to Find Solutions}

From actual production, we collect 300 data and divide them into 2 groups, the first group containing 150 data is used as training examples, and the other one is used as testing examples. We adopt the Gaussian function as a kernel function in PCM algorithms for improving SVDD, which is $K\left(x_{i}, x_{j}\right)=\exp \left(-\left\|x_{i}-x_{j}\right\|^{2} / \sigma^{2}\right)$. When we use the Gaussian function as the kernel function, we get $K(x, x)=1$ and $\left\|\phi\left(x_{k}\right)-\phi\left(v_{i}\right)\right\|^{2}=2-2 K\left(x_{k}, v_{i}\right)$.

Table 4 shows the defect diagnosis accuracy rate comparison of fibre gluing system between original SVDD multi-classifier and the improving SVDD multi-classifier by using PCM. We can see, by using our proposed new algorithm, the performance of the resulting classifier is improved significantly, and the accuracy is up to $97 \%$ which is much better than that of the original SVDD multi-classifier. We can conclude that PCM help SVDD multi-classifier overcome the negative effects of the outliers and noise, and the robustness and accuracy improved.

Table 4. The accuracy comparison of the 2 multi-classifier

\begin{tabular}{ll}
\hline Method & Accuracy rate \\
\hline Original SVDD & $82 \%$ \\
PCM based SVDD & $97 \%$ \\
\hline
\end{tabular}

Table 5. Status analysis of the gluing control system

\begin{tabular}{|c|c|c|c|c|c|}
\hline \multicolumn{5}{|c|}{ Inputs } & \multirow[t]{2}{*}{ outpu } \\
\hline $\mathrm{F} 1$ & $\mathrm{~F} 2$ & F3 & F4 & F5 & \\
\hline 0.60 & 0.75 & 0.67 & 0.48 & 0.5 & normal \\
\hline 0.66 & 0.75 & 0.22 & 0.78 & 0.5 & R6 \\
\hline 0.67 & 0.90 & 0.85 & 0.48 & 0.5 & $\mathrm{R} 2$ \\
\hline 0.65 & 0.74 & 0.64 & 0.48 & 0.5 & normal \\
\hline
\end{tabular}

Table 5 shows some defect diagnosis results, which is consistent with the actual status. When we get the physical data from the sensors respectively, and then input them into the PCM improved SVDD method, we can get the status of fibre gluing control system. The input section is the physical data which has 5 parameters, and the output section is the status of the fibre gluing system and has 6 statuses which include 5 defect statuses plus 1 normal. Because the defect diagnosis serving in the fibre gluing system gives signals to the control system, the stability of the control system based TRIZ is enhanced, and the production quality is improved. 


\section{Conclusions}

This paper describes work to integrate SVDD and TRIZ techniques to produce a method combining the best features of both. The paper also describes the research on the defect diagnosis method of the fibreboard gluing control system. Since TRIZ can expedite design process and help designer solve problems in a creative mode, the paper analyzed the supplying glue system problem, formulated the mini-problem and defined the conflicting elements, got the inventive principle to solve the contradict problem.

\section{Acknowledgment}

This topic of research is supported by the Basic National Project Foundation of China (Program No. 2008IM030400).

\section{References}

1. Domb, E.: QFD and TIPS/TRIZ. In: Proceedings of the 3rd International Symposium on QFD, Linkoping, Sweden (1997)

2. Yamashina, H., Ito, T., Kawada, H.: Innovative product development process by integrating QFD and TRIZ. International Journal of Production Research (November 2010)

3. Altshuller, G.: Creativity as an exact science:the theory of the solution of inventive problems, pp. 10-14. Gordon and Breach, New York (1995)

4. Li, T.-S., Huang, H.-H.: Applying TRIZ and Fuzzy AHP to develop innovative design for automated manufacturing system. Expert Systems with Applications 36, 8302-8312 (2009)

5. Savransky, S.D.: Engineering of Creativity: Introduction to TRIZ Methodology of Inventive Problem Solving. CRC Press, Boca Raton (2000)

6. Altshuller, G.: Creativity as an exact science, Moscow (1979) (in Russian); translated into English by AnthonyWhite (1988)

7. Mann, D.: Axiomatic design and TRIZ: compatibilities and contradictions. In: Proceedings of ICAD 2002, Cambridge, MA, pp. 10-11 (June 2002)

8. Tan, R.H.: Theory of Inventive Problem Solution. Science Press, China (2004)

9. Tax, D.M.J., Duin, R.P.W.: Uniform object generation for optimizing one-class classifiers. J. Mach. Learn. Res. 2, 155-173 (2001)

10. Lee, D., Lee, J.: Domain described support vector classifier for multi-classification problems. Pattern Recognition 40, 41-51 (2007)

11. Hu, Q., He, Z., Zhang, Z., Zi, Y.: Fault diagnosis of rotating machinery based on improved wavelet package transform and SVMs ensemble. Mechanical Systems and Signal Processing 21, 688-705 (2007)

12. Podsiadlo, P., Stachowiak, G.W.: Fast classification of engineering surfaces without surface parameters. Tribology International 39, 1624-1633 (2006) 